\title{
On the Effectiveness of Symmetry Breaking
}

\author{
Russell Miller ${ }^{1}$, Reed Solomon ${ }^{2}$, and Rebecca M. Steiner ${ }^{3}$ \\ 1 Queens College and the Graduate Center of the City University of New York \\ Flushing NY 11367 \\ 2 University of Connecticut \\ Storrs CT 06269 \\ 3 Vanderbilt University \\ Nashville TN 37240 \\ r.m.steiner@vanderbilt.edu
}

\begin{abstract}
Symmetry breaking involves coloring the elements of a structure so that the only automorphism which respects the coloring is the identity. We investigate how much information we would need to be able to compute a 2-coloring of a computable finite-branching tree under the predecessor function which eliminates all automorphisms except the trivial one; we also generalize to $n$-colorings for fixed $n$ and for variable $n$.
\end{abstract}

\section{Introduction}

Symmetry has always been a crucial concept in mathematics. We think of symmetry as a geometric property, but in fact, symmetries appear in many other branches of math as well. The symmetries of a mathematical structure are precisely its automorphisms - the bijections from the structure onto itself which preserve the essential properties of the structure. Some structures have many symmetries, and others have only one (the identity, or trivial automorphism).

Symmetry breaking involves coloring the elements of a structure in such a way that the only automorphism which respects the coloring is the trivial one; "breaking" symmetries can be thought of as "killing off" automorphisms.

Definition 11 An $n$-coloring of a structure is a function from the domain of the structure into a set of size $n$. It is said to distinguish the structure if there are no nontrivial automorphisms of the structure which respect the equivalence relation defined by the coloring. If a distinguishing $n$-coloring exists, then the structure is said to be $n$-distinguishable.

Definition 12 The distinguishing number of a structure is the smallest $n \in \omega$ such that the structure has a distinguishing $n$-coloring. If it exists, then the structure is finitely distinguishable.

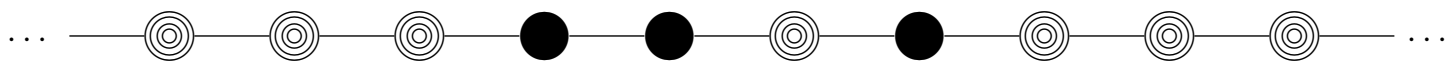

As an example, consider a graph that looks like the integers, as shown here. As a graph, it has infinitely many automorphisms. But there is a way to color the elements of this graph with just two 
colors so that the only automorphism which respects the coloring is the trivial one. A certain three elements are given the "solid" color, as in the figure here, while the rest are given the "striped" color, and the only symmetry of this graph which respects this coloring is the identity. So we would say that this graph has distinguishing number 2 .

Symmetry breaking has been studied extensively by combinatorists, with very recent results detailed in [1], [5], and [6]. In fact, the work found in the next section on symmetry breaking from a computability-theoretic perspective was inspired by a result from one of these articles:

Theorem 13 ([1], Theorem 3.1) The countable random graph has distinguishing number 2 .

This is extremely surprising. The random graph has continuum-many automorphisms, and is ultrahomogeneous: every finite partial automorphism extends to an automorphism of the entire graph. (This says that, in a certain sense, its automorphisms are dense, within the finite partial maps respecting its edge relation.) Moreover, while the result of Theorem 13 was not at all intended to be an effectiveness result, the construction of the distinguishing coloring of the countable random graph in [1] is indeed effective in the edge relation of the graph. In other words, a computable copy of the random graph has a computable distinguishing 2-coloring.

Knowing that this holds for the random graph inspired us to investigate the same question for other structures: what kinds of computable structures have computable distinguishing $n$-colorings? It was this question which led to our study of effective symmetry breaking in computable finitebranching predecessor trees, which form a natural first step in the subject, mainly because the automorphisms of such structures are readily understood.

Definition 14 A tree is a partial order $\prec$ on a set $\mathcal{T}$ of nodes, with a least element $r$ (the root) under $\prec$, such that for every $x \in T$, the set $\{y \in T: y \prec x\}$ is well-ordered by $\prec$. If every chain under $\prec$ has order type $\leq \omega$ (that is, if the tree has height $\leq \omega$ ), then each $x \in T$ has an immediate predecessor under $\prec$. A predecessor tree is a tree of height $\leq \omega$ in a language with equality and one unary function $P$, the predecessor function, for which $P(r)=r$ and $P(x)$ is the immediate predecessor of $x$ whenever $x \neq r$. If $\mathcal{T}$ has domain $\omega$ and $P$ is computable, we call $\mathcal{T}$ a computable predecessor tree; such $\mathcal{T}$ correspond precisely to computable subtrees of the tree $\omega^{<\omega}$ of finite strings from $\omega$. (The underlying partial order on such a $T$ is computable from $P$, although not definable by finitary formulas using $P$.)

A predecessor tree is finite-branching if, for every $y$, there are only finitely many $x \in T$ with $P(x)=y$.

Trees which are computable as partial orders (but for which the predecessor function is not necessarily computable) are considered in a different context in $[3,4]$, which may provide useful background for readers interested in investigating these questions. In such a tree, it is not generally possible to compute the level of a node, and this makes it substantially more difficult to determine which nodes lie in the same orbit under automorphisms of the tree. It would be natural to attempt to extend the results of this article to computable trees under $\prec$. Some previous effectiveness results about predecessor trees appear in [7], while for more general effectiveness results about symmetries as automorphisms, we refer the reader to [2].

\section{The Effectiveness Results}

The most natural question to address first is whether a computable finite-branching predecessor tree with distinguishing number 2 must have a computable distinguishing 2-coloring. 
Theorem 21 There is a computable finite-branching predecessor tree which is distinguished by a 2-coloring but not by any computable 2-coloring.

Proof. We will build our tree $\mathcal{T}$ in such a way that no (partial) computable function $\varphi_{e}$ can be a distinguishing 2-coloring of the tree. We start by describing the basic module - the strategy which will guarantee that for some fixed $e, \varphi_{e}$ is not a distinguishing 2-coloring of the tree. We build a finite tree $\mathcal{T}_{e}$ beginning with a root, five immediate successors of the root, and one immediate successor each for four of those five immediate successors of the root, as shown here.

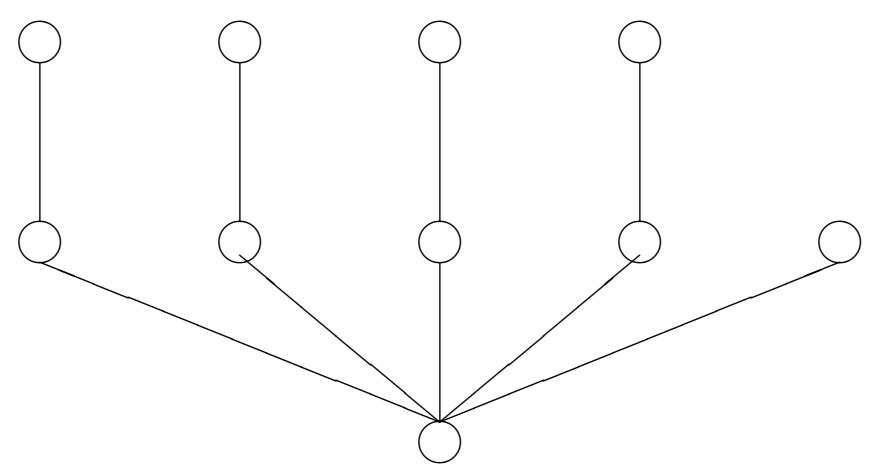

We wait for $\varphi_{e}$ to converge on each of these ten inputs. If $\varphi_{e}$ doesn't converge on all the inputs or converges outside the set $\{0,1\}$, then we do nothing further, because $\varphi_{e}$ is not a 2-coloring of $\mathcal{T}_{e}$. If $\varphi_{e}$ converges on all ten nodes to values in $\{0,1\}$ in such a way that there is already a nontrivial automorphism of $\mathcal{T}_{e}$ which preserves the coloring, then we do nothing further, because $\varphi_{e}$ is not a distinguishing coloring of $\mathcal{T}_{e}$. So, without loss of generality, suppose $\varphi_{e}$ converges on all ten nodes to values in $\{0,1\}$ and colors them as shown.

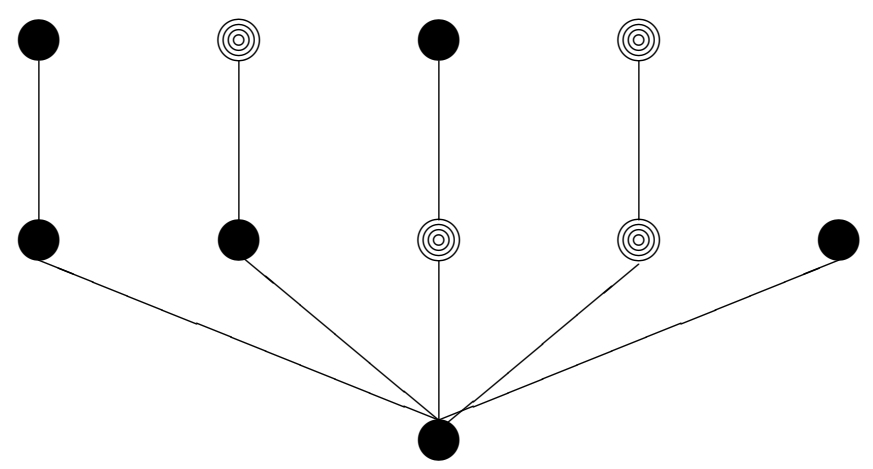

We do not want $\varphi_{e}$ to be a distinguishing coloring of $\mathcal{T}_{e}$, so we respond by adding three more nodes to $\mathcal{T}_{e}$, as seen below. (For convenience, nodes are now identified as in $\omega^{<\omega}$.) 


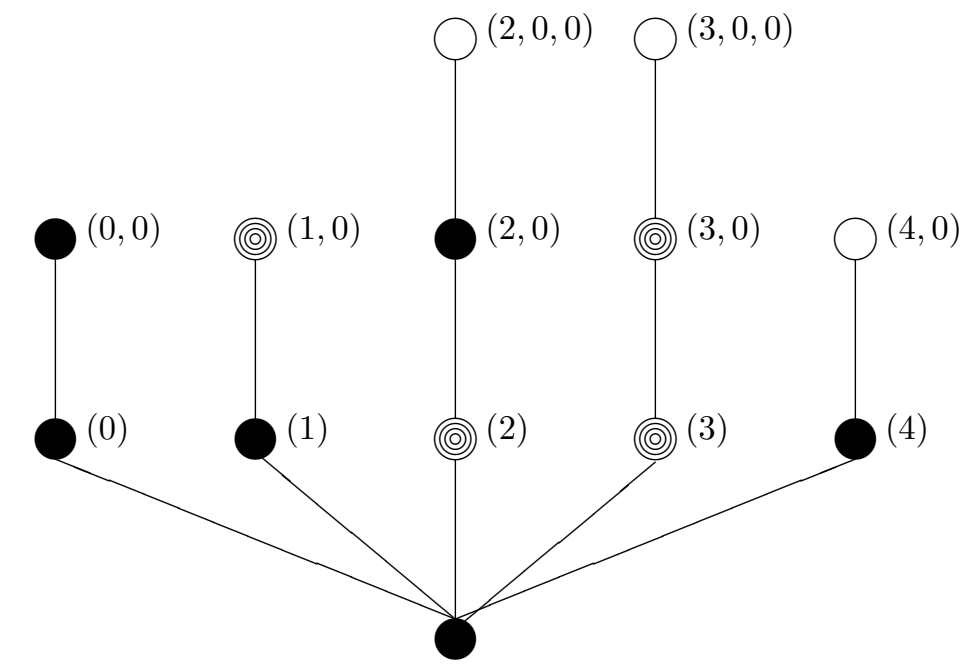

We have now made it impossible for $\varphi_{e}$ to be a distinguishing 2-coloring of $\mathcal{T}_{e} \cdot \varphi_{e}$ must color the new node $(4,0)$ at level 2 , and whichever color it chooses, there will be a nontrivial automorphism of $\mathcal{T}_{e}$ which respects that coloring. However, there does exist another 2-coloring $f_{e}$ which distinguishes $\mathcal{T}_{e}$ : change (4) to a striped node, while keeping the other colors and coloring the remaining three nodes with either color. Under this coloring the tree is rigid.

We put these basic modules together to build one big tree $\mathcal{T}$ as follows. Start with a spine: a single path $d_{0}<d_{1}<d_{2}<\cdots$ of nodes, among which $d_{0}$ will be the root of $\mathcal{T}$. Above each $d_{2 e+1}$, in addition to $d_{2 e+2}$, we place a node $r_{e}$. Then we build a copy of the tree $\mathcal{T}_{e}$ with $r_{e}$ as its root. Thus no $\varphi_{e}$ is a distinguishing 2-coloring of $\mathcal{T}$, but $\mathcal{T}$ is distinguishable by combining the 2-colorings $f_{e}$ for each $T_{e}$ into a single $f$ and coloring every node on the spine striped. The spine is fixed by every automorphism of $T$, as is each $r_{e}$, and this (noncomputable) $f$ then ensures that no automorphism except the identity can respect $f$.

In the tree constructed in Theorem 21, the branching function is not computable: it was not decidable which of the $(4,0)$ nodes (in all the different finite subtrees $\mathcal{T}_{e}$ ) have successors and which do not. So one naturally asks whether a computable finite-branching tree with distinguishing number 2 and with computable branching function would necessarily have a computable distinguishing 2coloring. However, the answer is still no: below, in Theorem 23, we construct such a tree with no computable distinguishing 2-coloring.

We start by describing the balanced 2 -coloring of the complete binary tree $2^{<\omega}$. Two nodes in this tree are called siblings if they are of the form $\sigma^{\wedge} 0$ and $\sigma^{\wedge} 1$, that is, if they have the same immediate predecessor $\sigma$. Thus every node except the root has exactly one sibling. A 2-coloring is balanced if it colors each node differently from its sibling: for instance, every node $\sigma^{\wedge} 0$ is solid and every node $\sigma^{\wedge} 1$ is striped. This example is isomorphic to every other balanced 2-coloring of $2^{<\omega}$, except for the color of the root. We will therefore speak of the balanced 2-coloring with striped root or the balanced 2-coloring with solid root. It is clear, by induction on the lengths of nodes, that each 
balanced 2-coloring distinguishes $2^{<\omega}$, i.e., no automorphism of $2^{<\omega}$ except the identity respects this coloring. Likewise, we speak of balanced 2-colorings of finite binary trees $2^{n}$.

However, it is also possible for an unbalanced 2-coloring to distinguish $2^{<\omega}$. As an example, color the nodes so that every node $1^{n}$ is striped, and also every node $1^{n} 0$ is striped, with all other nodes colored according to the balanced coloring with striped root. The two siblings 0 and 1 at level 1 are both striped, but the two successors of 0 are two different colors, while those of 1 are both striped. Therefore no automorphism respecting the coloring can interchange 0 with 1 , and one then uses this same argument to go upwards through all levels of the tree and see that each level is fixed pointwise by every automorphism respecting the coloring.

There are in fact many of these unbalanced colorings. However, once an imbalance has been introduced, it perpetuates itself.

Lemma 1. In a distinguishing 2 -coloring of $2^{<\omega}$, if some siblings $\sigma^{\wedge} 0$ and $\sigma^{\wedge} 1$ share a color, then either some two siblings extending $\sigma^{\wedge} 0$ share a color as well, or else some two siblings extending $\sigma^{\wedge} 1$ share a color.

Proof. If not, then the coloring would restrict to the balanced coloring on the tree above $\sigma^{\wedge} 0$, and also on the tree above $\sigma^{\wedge} 1$, with the same colored root in both. Therefore, there would be an automorphism interchanging $\sigma^{\wedge} 0$ with $\sigma^{\wedge} 1$ and respecting the coloring.

Corollary 22 For every finite binary tree $2^{<n}$, no unbalanced 2-coloring is distinguishing.

Proof. The reasoning is the same as in the lemma: any imbalance forces there to be another imbalance above itself. However, now this yields a pair of siblings with the same color at the very top level of the tree, and the automorphism which interchanges this pair and fixes all other nodes respects the unbalanced coloring.

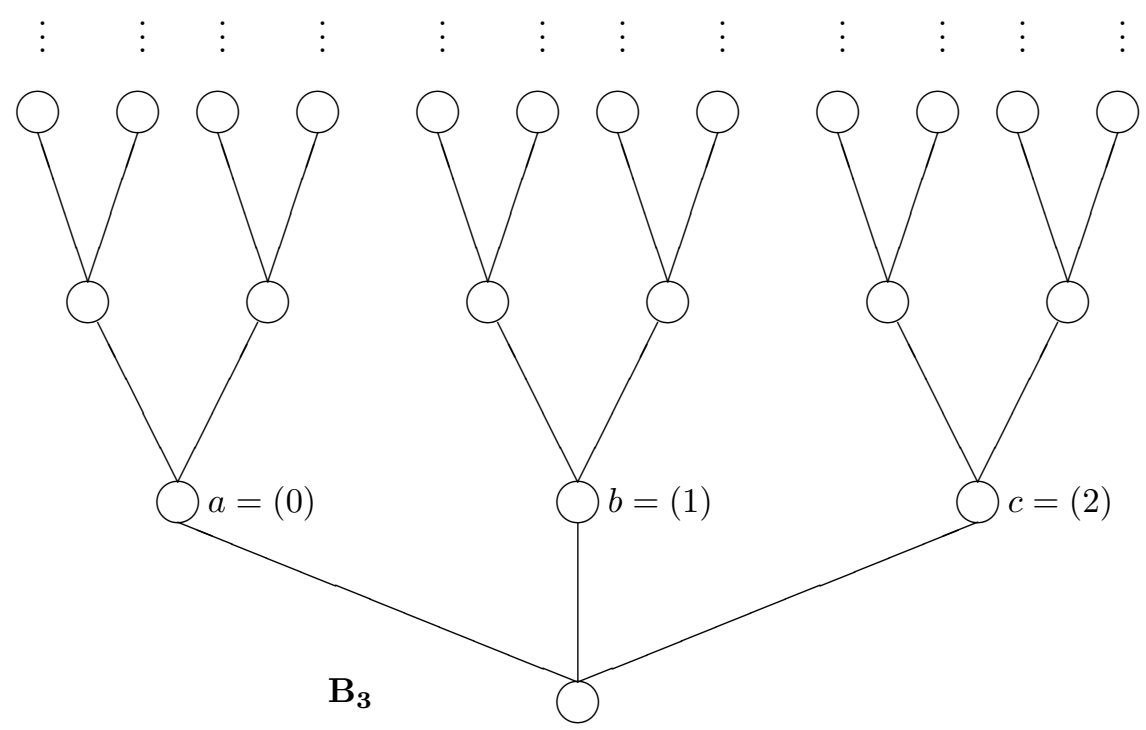

With these unbalanced colorings, we see that the tree $B_{3}$ in the figure above is 2-distinguishable:

$$
B_{3}=\left\{\sigma \in \omega^{<\omega}: \sigma(0)<3 \& \sigma(n)<2 \text { for } 0<|\sigma| \leq n\right\} .
$$


Indeed, $B_{3}$ has a computable distinguishing 2-coloring: just give the balanced coloring with solid root on the binary tree above $a$, the balanced coloring with striped root on the binary tree above $b$, and any unbalanced coloring (say with striped root) on the binary tree above $c$. We also have a distinguishing 2-coloring of each tree $B_{3, n}$ :

$$
B_{3, n}=B_{3}-\left\{\sigma \in B_{3}: \sigma(0) \neq 0 \&|\sigma| \geq n\right\}
$$

This $B_{3, n}$ is the tree gotten by chopping off (at level $n$ ) two of the three binary trees in $B_{3}$. To get a distinguishing 2-coloring of $B_{3, n}$, however, one is forced by the corollary above to use balanced colorings, with roots of different colors, on each of the finite trees above $b$ and $c$. The binary tree above $a$ is still complete, however: one can color it using the balanced 2-coloring with either color for the root, or using any unbalanced distinguishing 2-coloring.

Theorem 23 There is a computable finite-branching predecessor tree with computable branching function which is distinguished by a 2-coloring but not by any computable 2-coloring.

Proof. With the above observations, we can produce a tree $\mathcal{T}_{e}$ with distinguishing number 2 for which a given partial computable function $\varphi_{e}$ is not a distinguishing 2-coloring. Moreover, $\mathcal{T}_{e}$ will have computable branching (uniformly in $e$ ). This will form the basic module of the construction below. To build $\mathcal{T}_{e}$, start with three distinct immediate successors $a, b, c$ of the root node $r$, and begin building a copy of $2^{<\omega}$ above each, exactly as in the tree $B_{3}$. When we add level $s$ to these binary trees, we check to see whether $\varphi_{e, s}$ has converged yet on all three nodes at level 1 . If it never does so, or if it gives values $\notin\{0,1\}$ for any of them, then we simply keep building a copy of $B_{3}$. However, if it does output values in $\{0,1\}$ for all three, then we change our strategy. Without loss of generality, say that $\varphi_{e}(b)=\varphi_{e}(c)$. Once we see this, we end the construction of the binary trees above $b$ and $c$ : they are complete up to level $s$, but contain no nodes at all above level $s$. (Above $a$, we continue to build the complete binary tree, although in fact putting a single node at level $n+1$ above $a$ would suffice.)

The point is that, having committed to the same color for both $b$ and $c, \varphi_{e}$ is now trapped into giving a non-distinguishing 2-coloring of $\mathcal{T}_{e}$. By the Corollary, the only way to give a distinguishing 2-coloring above $b$ is to give the balanced 2-coloring, up to level $n$; and the same above $c$. However, then there will be an automorphism of $\mathcal{T}_{e}$ interchanging $b$ with $c$ and respecting this coloring, so $\varphi_{e}$ failed to distinguish $\mathcal{T}_{e}$ by its coloring.

On the other hand, $\mathcal{T}_{e}$ is 2-distinguishable, exactly as above: just color $b$ and $c$ different colors, and then use the balanced coloring above each of them, while coloring the complete binary tree above $a$ with any distinguishing 2 -coloring of $2^{<\omega}$. (Clearly no automorphism of this $\mathcal{T}_{e}$ can avoid fixing $a$, so the choice between balanced and unbalanced above $a$ is irrelevant.)

Finally, we wish to combine these basic modules to build a single computable tree $\mathcal{T}$, with computable finite branching, which has distinguishing number 2 but has no computable distinguishing 2-coloring. This is straightforward. Start with a spine $d_{0}<d_{1}<d_{2}<\cdots$, among which $d_{0}$ will be the root of $\mathcal{T}$. Above each $d_{2 e+1}$, in addition to $d_{2 e+2}$, we place a node $r_{e}$. Then we build a copy of the tree $\mathcal{T}_{e}$ with $r_{e}$ as its root, diagonalizing against the possible coloring $\varphi_{e}$ exactly as above in Theorem 21, using the three successors $a_{e}, b_{e}$, and $c_{e}$ of $r_{e}$. No $\varphi_{e}$ can be a distinguishing 2-coloring of the entire tree $\mathcal{T}$, because, assuming $\varphi_{e}$ is total, there will be some nontrivial automorphism of $\mathcal{T}_{e}$ which respects the coloring $\varphi_{e}$, and this automorphism extends to an automorphism of all of $\mathcal{T}$ just by fixing the rest of $\mathcal{T}$ pointwise. 
The branching function of a computable finite-branching predecessor tree is always $\mathbf{0}^{\prime}$-computable. However, it turns out that even with a $\mathbf{0}^{\prime}$-oracle, we could not necessarily compute a distinguishing 2-coloring of such a tree with distinguishing number 2 .

Theorem 24 There is a computable finite-branching predecessor tree which is distinguished by a 2-coloring but not by any $\mathbf{0}^{\prime}$-computable 2 -coloring.

Proof. This proof uses a simple modification to the trees $\mathcal{T}_{e}$ used to build $\mathcal{T}$ in Theorem 23. Now that the branching is allowed to be noncomputable, we may temporarily stop building the tree $\mathcal{T}_{e}$ at levels $>n$ above $b$ and $c$ (when $\varphi_{e}$ has given the same color to $b$ and $c$ ), and then resume building the complete binary tree above $b$ and $c$ when/if $\varphi_{e}$ "changes its mind" about its coloring of $b$ and $c$. This makes the branching (above these nodes at level $n$ ) noncomputable, since we do not know whether we will ever add nodes at level $n+1$. However, it enables us to satisfy the following requirement.

$$
\mathcal{R}_{e}: \lim _{t} \varphi_{e}(x, t) \text { is not a distinguishing } 2 \text {-coloring of } T_{e} .
$$

These requirements together will show that $\mathcal{T}$ has no $\mathbf{0}^{\prime}$-computable distinguishing 2-coloring, where $\mathcal{T}$ is built from the trees $\mathcal{T}_{e}$ exactly as before.

The alteration to the construction of $\mathcal{T}_{e}$ is simple. As before, wait for $\varphi_{e, s}(a, 0), \varphi_{e, s}(b, 0)$, and $\varphi_{e, s}(c, 0)$ to halt with values in $\{0,1\}$. Pick two of them which have the same value, and stop building the binary trees above those two nodes (while continuing to build a binary tree above the third node). Meanwhile, wait for $\varphi_{e, s}(a, 1), \varphi_{e, s}(b, 1)$, and $\varphi_{e, s}(c, 1)$ to halt with values in $\{0,1\}$. When and if this happens, these values supersede those from before: for example, if previously we had halted construction above $b$ and $c$ (as in the original description of $\mathcal{T}_{e}$ ), but now $\varphi_{e}(a, 1)=\varphi_{e}(b, 1)=0 \neq \varphi_{e}(c, 1)$, then we build up the trees above $b$ and $c$ until all three have the same height, then continue building the tree above $c$ but stop building the ones above $a$ and $b$. On the other hand, if $\varphi_{e, s}(a, 1)=\varphi_{e}(a, 0), \varphi_{e, s}(b, 1)=\varphi_{e}(b, 0)$, and $\varphi_{e, s}(c, 1)=\varphi_{e}(c, 0)$, then $\varphi_{e}$ has not changed its mind, and we do not resume construction above the two nodes above which it was stopped. We then continue on to consider $\varphi_{e}(a, 2)$, etc., using the same program relative to the values $\varphi_{e}(a, 1)$, etc., and so on for all $t$.

If $\lim _{t} \varphi_{e}(x, t)$ exists for all three values $x \in\{a, b, c\}$, then we wind up in the same situation as in the previous proof, showing that this limit cannot be a distinguishing 2-coloring of $\mathcal{T}_{e}$, so that $\mathcal{R}_{e}$ holds. On the other hand, if the limit fails to exist (but $\varphi_{e}$ is total with range $\subseteq\{0,1\}$ ), then we simply built $B_{3}$ above $r_{e}$, and $B_{3}$ is indeed 2-distinguishable (although not by $\lim _{t} \varphi_{e}(x, t)$ ). Finally, if $\varphi_{e}$ is not total or assumes values $>1$, then $\mathcal{R}_{e}$ will hold, and the $\mathcal{T}_{e}$ we build is either a copy of $B_{3}$ or a copy of some $B_{3, n}$, both of which are 2-distinguishable. (Technically, even if range $\left(\varphi_{e}\right)$ contains some values $>1$, the limit could still have values 0 and 1 only. However, if this holds, then some other $\varphi_{e^{\prime}}$ would have the same limit and would have range $\subseteq\{0,1\}$, so that $\mathcal{R}_{e^{\prime}}$ would have taken care of showing that $\lim _{t} \varphi_{e}$ was not a distinguishing 2-coloring.)

So how much information would we need to compute a distinguishing 2-coloring of a computable finite-branching predecessor tree with distinguishing number 2? We answer this below in Theorem 25 , but we begin by defining an extendible node of a tree to be a node which lies on an infinite path.

Lemma 2. If all nodes of a computable predecessor tree $T$ are extendible, then $T$ is 2 -distinguishable.

Proof. This is true even if the tree is infinite-branching, so we prove it for this more general case. Suppose we have a computable predecessor tree with every node extendible. Label the nodes at 
level 1 of the tree $x_{1}, x_{2}, \ldots$ in order of their enumeration into the tree. Color $x_{1}$ striped. Color $x_{2}$ solid, and color every node at level 2 above $x_{2}$ striped. Color $x_{3}$ solid, color every node at level 2 above $x_{3}$ solid, and color every node at level 3 above $x_{3}$ striped, and so on for $x_{4}, x_{5}, \ldots$. This procedure distinguishes each node at level 1 from every other node at level 1.

Fix an $n>0$, and consider the immediate (level-2) successors $y_{1}, y_{2}, \ldots$ of $x_{n}$. These may have already been colored by the previous instructions; in fact, their successors up to level $n$ will already be colored. Color the level- $(n+1)$ successors of $y_{1}$ striped, the level- $(n+1)$ successors of $y_{2}$ solid and its level- $(n+2)$ successors striped, then the same above $y_{3}$ with solid-solid-striped, and so on. When we do this for every $n$, each node at level 2 is distinguished from all its siblings at level 2.

We continue in this vein to distinguish each node at level $k$ from every other node at level $k$, for every $k$.

Here is the algorithm for determining the color of an arbitrary node on such a tree if we've used the above coloring:

Choose a node on the tree. Call it $n$. Call the root $r$.

( $\star$ ) Use the predecessor function to determine the level of $n$ above $r$. Call this level $l$.

Label the immediate successors of $r$ with $x_{1}, x_{2}, \ldots$

If $n$ sits above $x_{l}$, then $n$ is red.

Else, if $n$ sits above $x_{i}$ for some $i>l$, then $n$ is blue.

Else, $n$ sits above $x_{i}$ for some $i<l$. Let this $x_{i}$ be the new $r$, and go back to $(\star)$.

Theorem 25 If a computable finite-branching predecessor tree has distinguishing number 2, then it has a $\mathbf{0}^{\prime \prime}$-computable distinguishing 2-coloring.

Proof. Because the tree is finite-branching, with a $\mathbf{0}^{\prime \prime}$-oracle we can determine, for each immediate successor $y$ of a given node $x$, whether $y$ is extendible or not: König's Lemma states that a nonextendible node must have only finitely many nodes extending it. Above the extendible ones we use the process illustrated above in Lemma 2. For each extendible $x$, consider the (finite) subtree containing $x$, the non-extendible immediate successors of $x$ and all of their successors. There must be a way to distinguish this subtree with a 2-coloring, since the tree has a distinguishing 2-coloring. Each non-extendible node has only finitely many nodes above it. With the $\mathbf{0}^{\prime \prime}$-oracle we can find them all, and we can try out all the possible colorings until we find one which admits no non-trivial automorphism. Having done all of this, we know that every automorphism of the tree which respects our coloring and which fixes $x$ must also fix each immediate successor $y$ (and all the nodes above each non-extendible $y$ ). By induction on levels, this means that an automorphism which respects the coloring must fix each single node, i.e. must be the identity.

Thus the existence of a distinguishing 2-coloring is equivalent to the existence of such a coloring computable in $\mathbf{0}^{\prime \prime}$. This significantly reduces the complexity of the property (for computable finitebranching trees $\mathcal{T}$ under predecessor) of being 2-distinguishable. On its face, that property was $\Sigma_{2}^{1}$ : it said that there exists a function (the coloring) such that every automorphism of $\mathcal{T}$ either fixes all nodes or disrespects the coloring. Of course, this complexity can quickly be reduced, since the complexity of an orbit in a finite-branching computable predecessor tree is at most $\Pi_{2}^{0}$. Nevertheless, 2-distinguishability still could have been $\Sigma_{1}^{1}$-hard for these trees, up until we established Theorem 25 , which showed that we need only quantify over $\mathbf{0}^{\prime \prime}$-computable functions, not over all functions, to define 2-distinguishability.

We now investigate how much further we can lower the complexity of the property of 2distinguishability, and whether the same complexity level holds for $n$-distinguishability. Theorem 
26 answers these questions. Subsequently we will consider distinguishability by finite colorings, i.e., colorings with finitely many colors, but with no fixed bound on the number of colors.

Theorem 26 For each fixed $n$, the property of having a distinguishing n-coloring is $\Pi_{2}^{0}$-complete within the class of finite-branching predecessor trees.

Proof. We will show that having a distinguishing 2-coloring is $\Pi_{2}^{0}$-complete, and we will explain how the argument extends to an $n$-coloring for fixed $n$.

A finite-branching predecessor tree has no distinguishing 2-coloring if and only if

$$
\exists \sigma_{1}, \ldots, \sigma_{k}\left[\begin{array}{l}
\sigma_{1}, \ldots, \sigma_{k} \text { are not extendible and have a common predecessor } \tau \& \\
\text { the tree }\{\tau\} \cup \bigcup_{i=1}^{k}\left\{\delta: \delta \supseteq \sigma_{i}\right\} \text { has no distinguishing 2-coloring }
\end{array}\right] .
$$

Non-extendibility is a $\Sigma_{2}^{0}$ property, and the other two conjuncts inside the brackets are each computable. So, not having a distinguishing 2-coloring is $\Sigma_{2}^{0}$. Thus, having a distinguishing 2-coloring is $\Pi_{2}^{0}$. Notice that if "2-coloring" in the above argument were replaced with " $n$-coloring," the result would still hold; so, for fixed $n$, having a distinguishing $n$-coloring is $\Pi_{2}^{0}$.

To show completeness, we start by building a copy of the tree $B_{3}$ as follows. Begin with the root and the three nodes at level 1 . Then, whenever a new element is enumerated into the $e$-th c.e. set $W_{e}$, we add the whole next level of $B_{3}$ to our tree. If $W_{e}$ turns out to be finite, our copy of $B_{3}$ will only have finitely many levels, and thus will not be 2-distinguishable. If $W_{e}$ turns out to be infinite, then our $B_{3}$ will likewise be infinite, and thus 2-distinguishable. So the tree has a distinguishing 2-coloring just if $W_{e}$ is infinite.

If we define $B_{n+1}$ to be the tree whose root has exactly $(n+1)$ immediate successors and every other node has exactly $n$ immediate successors, then if we replace $B_{3}$ with $B_{n+1}$ in the immediately preceding paragraph, we show that having a distinguishing $n$-coloring for some fixed $n$ is $\Pi_{2}^{0}$-complete as well.

It remains to consider "having a distinguishing $n$-coloring for some (arbitrary) n." This is the property of being finitely distinguishable. Expressing this property takes an extra $\exists$ quantifier, so it is plausible that having a finite distinguishing coloring is $\Sigma_{3}^{0}$-complete.

Theorem 27 The property of having a distinguishing finite coloring is $\Sigma_{3}^{0}$-complete within the class of finite-branching predecessor trees.

Proof. Define $S_{\infty}$ to be the following tree: There is an infinite "spine," and the node $\alpha_{n}$ on the spine at level $n$ has exactly $n$ additional immediate successors, all of which are terminal. We will show, using the tree $S_{\infty}$, that being finitely distinguishable is $\Sigma_{3}^{0}$-complete by giving a 1-reduction from the set of indices for finitely distinguishable trees to the set Cof.

We start by building the tree $S_{\infty}$. Let $A_{k}$ be the subtree consisting of $\alpha_{k}$ and all its nonextendible immediate successors. We wait for elements to be enumerated into $W_{e}$. At stage $s$, suppose $m \in W_{e, s}-W_{e, s-1}$. Then we make $A_{m}$ rigid by adding paths of distinct finite lengths above the immediate successors of $\alpha_{m}$.

We claim that the resulting tree is finitely distinguishable if and only if $W_{e}$ is cofinite. Suppose $\overline{W_{e}}$ is finite and nonempty. (If $\overline{W_{e}}$ is empty, then the tree is rigid, i.e., 1-distinguishable.) Then every tree $A_{k}$ with $k>\max \left(\overline{W_{e}}\right)$ is rigid. Thus the whole tree is $\max \left(\overline{W_{e}}\right)$-distinguishable. Now suppose $\overline{W_{e}}$ is infinite. Then, given any $n$, there is $A_{k}$ with $k>n$ for which $A_{k}$ is $k$-distinguishable but not $n$-distinguishable. Thus, for each $n$, the whole tree is not $n$-distinguishable. So the tree has a finite distinguishing coloring just if $W_{e}$ is cofinite. 


\section{$3 \quad$ Further Questions}

The most natural next question is:

Question 1. What are the analogous results for computable infinite-branching predecessor trees?

We have already begun answering this question, and results will be forthcoming in an expansion of this abstract.

In [7], we showed that many computability-theoretic results which were true of finite-branching predecessor trees were also true of finite-valence pointed graphs, and so we also ask:

Question 2. Do any of these results carry over to computable finite-valence pointed graphs?

This is our expected next step after considering the infinite-branching predecessor trees.

\section{References}

1. W. Imrich, S. Klavzar, \& V. Trofimov; Distinguishing infinite graphs, Electronic Journal of Combinatorics, 14 (2007), \#R36.

2. V. Harizanov, R. Miller, \& A. Morozov; Simple structures with complex symmetry, Algebra and Logic 49 (2010), 51-67.

3. S. Lempp, C. McCoy, R.G. Miller, \& R. Solomon; Computable categoricity of trees of finite height, Journal of Symbolic Logic $\mathbf{7 0}$ (2005), 151-215.

4. R.G. Miller; The computable dimension of trees of infinite height, Journal of Symbolic Logic 70 (2005), $111-141$.

5. S. M. Smith \& M. E. Watkins; Bounding the distinguishing number of infinite graphs, submitted for publication.

6. S. M. Smith, T. W. Tucker, \& M. E. Watkins; Distinguishability of infinite groups and graphs, Electronic Journal of Combinatorics 19 (2012), \#R27.

7. R. M. Steiner; Effective algebraicity, Archive for Mathematical Logic 52 (2013), 91-112. 